\title{
Unified viscoplasticity modelling and its application to fatigue-creep behaviour of gas turbine rotor
}

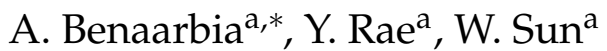 \\ ${ }^{a}$ Department of Mechanical, Materials and Manufacturing Engineering, University of \\ Nottingham, Nottingham, Nottinghamshire, NG7 2RD, UK
}

\begin{abstract}
This paper presents an elasto-visco-plastic finite element modelling framework including the associated UMAT codes to investigate the high temperature behaviour of gas turbine rotor steels. The model used in the FE study is an improved and unified multi-axial Chaboche-Lemaitre model which takes into account non-linear kinematic and isotropic hardening. The computational methodology is a three-dimensional framework following an implicit formulation and based on a radial return mapping algorithm. The UMAT is calibrated and validated across isothermal hold-time cyclic tests. The methodology developed is applied to a classical industrial gas turbine rotor where the study focuses its attention on the deformation heterogeneities and critical high stress areas within the rotor structure. The effect of thermal transients and geometry singularities on the development of residual stresses is underlined. Finally, the potential improvements and extensions of such FE viscoplastic analysis is discussed.
\end{abstract}

Keywords: Cyclic viscoplasticity, finite element, return mapping, gas turbine rotor

${ }^{*}$ Corresponding author 


\section{Introduction}

The developments in gas turbine construction technology and improvement of fuel efficiency rely on the use of high temperature materials suitable for service environment. During the life of a gas turbine it is exposed to high temperatures and repeated mechanical loads. These extreme operating conditions result in a variety of solicitations such as thermomechanical fatigue and fatigue-creep (and/or relaxation) affecting gas turbine components. Industrial gas turbines are extensively used at power plants designed for intermittent electricity generation, as a cost effective way of fulfilling the energy demand during peak periods. As power plants where gas turbines are used often need to operate in a flexible way, a requirement for the modelling of cyclic loading arises. The turbine rotor is a critical part of a gas turbine and is generally affected by various degradation modes, which could potentially lead to failure and downtime. To account for the damage resulting from these various solicitations, it is important to find out the magnitude of transient stresses generated.

Superalloys have been developed in 1940s and were found to perform very well at high temperatures. Their superior properties at elevated temperatures such as strength and creep resistance made them a material of choice for turbine rotors. In GTs, high pressure (HP) and low pressure (LP) turbine rotors operate in different environments, so their material requirements differ. HP turbine rotors operate in high temperature environments and LP turbine rotors operate in low temperature environments, therefore there is a danger of brittle fracture especially in the final stages of the turbine rotor. The last stage blades are the largest and witness high centrifugal stresses, therefore the main requirements are high proof stress and low fracture transition temperature. Typical materials fitting those requirements would be low alloy martensitic Ni-Cr-Mo-V steels strengthened with carbide, which stay ductile after hundreds of thousands of hours of operation. A 
commonly used grade is $26 \mathrm{NiCrMoV11-5.} \mathrm{Chromium} \mathrm{is} \mathrm{added} \mathrm{for} \mathrm{oxid-}$ ation and corrosion resistance. Molybdenum improves strength at high temperatures. Vanadium is a very strong carbide former and improves mechanical properties by increasing hardness and toughness. The material information used in the model was obtained from a range of experimental tests.

The rotor loading pattern consists of loading-unloading solicitations followed by long dwell periods at high range of temperatures. This involves creep and plasticity mechanisms, and interactions between them complicate modelling of the fatigue life. Within the last several decades a significant progress has been made in the development of the viscoplastic constitutive equations. Viscoplasticity models can predict both creep and cyclic plasticity which makes them well fit for the purpose. The modelling of plasticity and creep can be split into two broad groups, unified and non-unified theories (e.g. Perzyna [35], Olszak and Perzyna [34], Zienkiewicz and Cormeau [50], Bodner and Partom [9], Miller [29], Chaboche [13], Chaboche et al. [17], Cernocky and Krempl [12], Chaboche and Rousselier [16], Johnson and Cook [24], Contesti and Cailletaud [19], Kawai and Ohashi [25], Chaboche and Nouailhas [15], Chan et al. [18], Ohno [31], Tong et al. [43], etc.). In unified constitutive models, creep (viscous) and plastic strains are represented by a single inelastic measure and considered to be rate-dependent (e.g. Chaboche [14], Barrett et al. [5], Wang et al. [45], Ahmed et al. [1], Zhang and Xuan [48], etc.). While, the nonunified models consider creep strain to be rate-dependent but plastic strain on the other hand to be rate-independent, and look at them as two separate measures (e.g. Cailletaud and Sai [11], Velay et al. [44]). Non-unified models lack the ability of predicting some of the material behaviours such as ratcheting and interaction between creep and plasticity (see. Zienkiewicz and Cormeau [50], Corum and Blass [20], Krempl [27]). They are difficult to 
implement into finite element code due to the different flow rules involved.

A lot of the models have been developed based on the Prager [36] linear kinematic hardening model and Armstrong and Frederick [3] non-linear kinematic hardening model. It was demonstrated by Chaboche et al. [17] that if several kinematic hardening rules by Armstrong-Frederic are superimposed, the cyclic response simulation is greatly improved. Since then a variety of authors proposed their versions of the viscoplasticity models based on non-linear kinematic hardening rules (e.g., Ohno [31], Freed et al. [21], Ohno and Wang [32, 33], Freed and Walker [22], Arnold and Saleeb [4], Shutov and Kreibig [41], etc.), several of them have been reviewed by Besson [8] and Chaboche [14]. However, only a few of the models were applied to situations involving thermo-mechanical fatigue (TMF) (e.g., Zhang et al. [49], Barrett et al. [5]). There is also a very limited amount of information available in literature from multiaxial cases (see. Cailletaud et al. [10]). The most popular model is the viscoplastic constitutive theory developed by Chaboche, it was reviewed by Ambroziak and Klosowski [2] who concluded that it can be applied to a wide range of problems. Its applicability to modelling of turbine rotor geometries and materials was demonstrated by Zhan and Tong [47], Wang et al. [46], and Nayebi et al. [30] that used it for TMF damage assessment. In the unified viscoplasticity framework, the viscosity function dictates the viscoplastic strain rate. It has been shown by Becker and Hackenberg [7] that there is a high degree of non-linearity in the relationship between stress and inelastic strain. Therefore, a hyperbolic sine-function (e.g., [26]), power function (e.g., [40]) or exponential law (e.g., [37]) is required to represent the viscous response in an accurate manner.

The model used in this study is an extended version of the original Chaboche constitutive model, that was previously validated using experimental res- 
ults in several papers (e.g., Kyaw et al. [28], Barrett et al. [6], Saad et al. [39]). At maximum loads, temperatures within the rotor can be as high as $600{ }^{\circ} \mathrm{C}$ resulting in creep. The need for rate dependency arises from the conditions in which the rotor operates. A novel isotropic hardening law which takes into account cyclic softening is adopted. The kinematic evolution law based on the original Armstrong-Frederick type model is also considered. As turbine rotor undergoes anisothermal loading its material properties vary. This influence of temperature on the material parameters is taken into consideration in the model. The theoretical formulation of the constitutive model with 11 model parameters is based on thermodynamics of irreversible processes and is solved numerically using a return mapping algorithm. A transient thermo-mechanical analysis of an axisymmetric gas turbine rotor geometry is performed in the ABAQUS finite element software and material model is added using the UMAT (user defined material model) code. The UMAT was calibrated and validated across isothermal hold-time cyclic tests.

The first part of this paper introduces the theoretical formulations and background of the constitutive elasto-viscoplastic model. This is followed by a description of the algorithm formulation. An uniaxial validation of the constitutive model is then presented. The application of the model to $\mathrm{FE}$ analysis of a gas turbine rotor is addressed where all FE steps are first described in details. The last part describes and discusses the physical significance of the results obtained from the FE analysis. Several conclusions, drawn from the findings, are finally put forward, along with discussions of limitations of the current model and some proposals regarding future investigations. 


\section{Theoretical formulation of the constitutive model}

In the following, all expressions defined below are formulated within Thermodynamics of Irreversible Processes framework (Germain et al. [23]). For readers interested in this theoretical framework, more details are given in Chaboche [14].

In the current work, we assume the small strain framework and that the total strain tensor $\varepsilon_{i j}$ is composed of two parts: a small recoverable (revers-

ible) elastic strain $\varepsilon_{i j}^{e}$ and a large irreversible viscoplastic strain $\varepsilon_{i j}^{v p}$ such that

$$
\varepsilon_{i j}=\varepsilon_{i j}^{e}+\varepsilon_{i j}^{v p}
$$

The elastic strain is related to the stress through the linear elastic constitutive model, where the Cauchy stress tensor $\sigma_{i j}$ is given in terms of the elastic strain tensor $\varepsilon_{k l}^{e}$ by

$$
\sigma_{i j}=\left(2 G I_{i j k l}^{d}+K \delta_{i j} \delta_{k l}\right) \varepsilon_{k l}^{e},
$$

where the constants $G$ and $K$ are the shear and bulk moduli and $I_{i j k l}^{d}$ the deviatoric part of the fourth-rank identity tensor $I_{i j k l}$.

The material investigated herein is assumed to be isotropic and the yield criteria to be independent of the hydrostatic pressure (only depends on the deviatoric components of stresses). This leads us to the following von Mises yield criterion given here as

$$
f(\sigma, \mathrm{X}, R ; T)=J_{2}-R(T, \gamma(T))-k(T),
$$

where $k(T)$ denotes the yield stress, $R(T, \gamma(T))$ the isotropic hardening stress (drag stress) with $\gamma$ the viscoplastic multiplier, and $J_{2}$ is the second 
invariant defined by $J_{2}=\sqrt{3 / 2\left(S_{i j}-X_{i j}^{d}\right)\left(S_{i j}-X_{i j}^{d}\right)}$, where $S_{i j}$ is the deviatoric part of Cauchy stress tensor $\sigma_{i j}$ and $X_{i j}^{d}$ the deviatoric part of the back stress $X_{i j}$.

The magnitude of viscoplastic strain rate $\dot{\varepsilon}_{i j}^{v p}$ can be determined from the viscoplastic flow rule (normality rule), and is given as follows

$$
\dot{\varepsilon}_{i j}^{v p}=\dot{\gamma} N_{i j}=\frac{3}{2} \dot{\gamma}\left(S_{i j}-X_{i j}^{d}\right) J_{2}^{-1}
$$

where $N_{i j}$ is the gradient of the yield function with respect to the stress tensor.

In the following, we adopt a Norton power viscosity function to define the viscoplastic multiplier rate such that

$\dot{\gamma}=\Xi_{v}\left(\dot{\gamma}, X_{i j}, R ; T\right)$; where $\Xi_{v}=\left(f Z(T)^{-1}\right)^{n(T)}$ if $f>0, \Xi_{v}=0$ if $f \leq 0$,

where $Z(T)$ and $n(T)$ are, respectively, the viscoplastic resistance and hardening exponent functions which are both temperature dependent.

The following Chaboche-type kinematic hardening rule (see. Chaboche [14]) with two backstress contributions is adopted

$$
X_{i j}=X_{i j}^{(1)}+X_{i j}^{(2)} ; X_{i j}^{(m)}(T)=\frac{2}{3} v^{(m)}(T) \xi^{(m)}(T) \chi_{i j}^{(m)}(T) ; m=1,2,
$$

where $v^{(m)}(T)$ and $\xi^{(m)}(T)$ are material parameters depending only on temperature. The term $\chi_{i j}^{(m)}$ is the associated internal variable of the conjugate variable $X_{i j}^{(m)}$ to which it is linearly related (see Eq. 6). This internal variable characterises the internal strain misfit coming from the plastic straining. The evolution rule of $\chi_{i j}^{(m)}$ was first proposed by Armstrong and Frederick 
[3] and is given as

$$
\dot{\chi}_{i j}^{(m)}(T)=\dot{\varepsilon}_{i j}^{v p}-v^{(m)}(T) \dot{\gamma}(T) \chi_{i j}^{(m)}(T) ; m=1,2 .
$$

This form introduces the evanescent strain memory during cyclic mechanical loading responsible for the Baushinger effects. After substitution of Eq. 6 into Eq. 7, the anisothermal kinematic hardening stress evolution rule can be written as follows

$$
\dot{X}_{i j}^{(m)}(T)=v^{(m)}(T)\left(\frac{2}{3} \xi^{(m)}(T) \dot{\varepsilon}_{i j}^{v p}-X_{i j}^{(m)}(T) \dot{\gamma}\right)+\mathrm{Y}(T) X_{i j}^{(m)}(T) \dot{T},
$$

where $Y^{(m)}(T)=\left[\left(v^{(m)}(T)\right)^{-1} v_{, T}^{(m)}(T)+\left(\xi^{(m)}(T)\right)^{-1} \xi_{, T}^{(m)}(T)\right]$ and $x_{, T}$ defines the partial derivative of $x$ according to temperature $T$. It is worth noting that the static recovery term is not be retained in the modelling. The relaxation durations considered hereafter are short and thus we assume that all recovery processes are of dynamic origins (they take place only during the deformation).

In order to take into account the experimentally observed non saturating cyclic softening, the following drag stress $R$ vs. the associated internal variable $r$ relationship is adopted

$$
R(T, \gamma(T))=\zeta(T) \kappa(T) r(T, \gamma(T))+H(T) \gamma(T),
$$

where $\zeta(T)$ and $\kappa(T)$ are two material parameters depending only on the temperature, while $H(T)$ specifies the linear evolution of drag stress. The rate evolution of the associated internal variable $r$ is given as follows

$$
\dot{r}(T, \gamma(T))=\left[1-\zeta(T) r(T, \gamma(T))-\kappa^{-1}(T) H(T) \gamma(T)\right] \dot{\gamma}(T) .
$$

After time derivation of the drag stress $R$ and by substituting Eq. 9 into Eq. 10, the anisothermal isotropic hardening stress evolution rule is ob- 
tained

$$
\begin{aligned}
\dot{R}(T, \gamma(T)) & =\left[\kappa(T)-R(T, \gamma(T))+\zeta^{-1}(T) H(T)\right] \zeta(T) \dot{\gamma}(T) \\
& +\left[\gamma(T) H_{, T}(T)+(R(T, \gamma(T))-H(T) \gamma(T)) \varrho(T)\right] \dot{T},
\end{aligned}
$$

where $\varrho(T)=\zeta^{-1}(T) \zeta_{, T}(T)+\kappa^{-1}(T) \kappa_{, T}(T)$.

\section{Algorithm formulation of the constitutive model}

The numerical implementation of the model is based on a return mapping algorithm scheme (Simo and Hughes [42]). The procedure is split in two parts: In the first part, the set of internal variables $\left(\varepsilon_{i j}^{v p}, r\right.$ and $\chi_{i j}^{(m)}$ with $m=1,2)$ of the material do not evolve and only generation of elastic strains is considered (elastic prediction). Thus, all these internal variables are kept fixed, while the initial guess for the stress is computed directly by the total strain tensor $\varepsilon_{i j}$. In the second part, the error in the stress is corrected by identifying the actual change in the internal variables (inelastic correction). Thus the total strain tensor is kept fixed and only $\varepsilon_{i j}^{v p}, r$ and $\chi_{i j}^{(m)}$ evolve. The return mapping algorithm is taken care of by the constitutive law algorithm.

Hereafter, we denote all quantities at time $t^{(q)}$ with a subscript $(q)$ while all quantities at time $t^{(q+1)}$ are denoted by a subscript $(q+1)$. Increments $\Delta t=t^{(q+1)}-t^{(q+1)}$ are left with no subscripts. Tensorial notation is dropped whenever the algorithm is introduced to reduce the complication of indices and subscripts. The procedure starts at time $t^{(q)}$ with knowledge of the stresses $\sigma_{i j}^{(q)}$ and the total strain increment $\Delta \varepsilon_{i j}$ passed to the UMAT file from the previous equilibrium iteration carried out by ABAQUS. The aim now is to find the updated stresses $\sigma_{i j}^{(q+1)}$, equivalent viscoplastic multiplier $\gamma^{(q+1)}$, viscoplastic strains $\varepsilon_{i j}^{(q+1)}$ and both hardening variables $\mathrm{X}_{i j}^{(q+1)}$ and $R^{(q+1)}$. 
Let's first start with the following expression, where the elastic predictor $\sigma_{i j}^{t r ;(q+1)}$ will be referred to as $\sigma_{i j}^{t r}$

$$
\sigma_{i j}^{(q+1)}=\sigma_{i j}^{t r}-C_{i j k l} \Delta \varepsilon_{k l}^{v p}=\sigma_{i j}^{t r}-\dot{\gamma}^{(q+1)} C_{i j k l} N_{k l}^{(q+1)} \Delta t,
$$

with $C_{i j k l}$ stands for the fourth order Hooke's elastic operator. As only the deviatoric part $S_{i j}$ of the stress tensor $\sigma_{i j}$ affects the plastic analysis merely due to the fact that the hydrostatic pressure remains constant $\left(I_{i j k l}^{d} S_{k l}^{(q+1)}=\right.$ $S_{i j}^{(q+1)}$ and incompressible plasticity $S_{k k}^{(q+1)}=0$ ), the following expression is obtained

$$
\left(\frac{2}{3} J_{2}^{(q+1)}+2 G \dot{\gamma}^{(q+1)} \Delta t\right) N_{i j}^{(q+1)}=\frac{2}{3} J_{2}^{t r} N_{i j}^{t r}
$$

This equation implies that

$$
N_{i j}^{(q+1)}=N_{i j}^{t r} \text { and } \frac{2}{3} J_{2}^{(q+1)}+2 G \dot{\gamma}^{(q+1)} \Delta t=\frac{2}{3} J_{2}^{t r} .
$$

Eq. 5 can be used to solve for the viscoplastic multiplier $\gamma^{(q+1)}$. Rearranging this equation when $f>0$, and using Eq. 14 leads to

$$
\begin{aligned}
\Phi^{(q+1)} & =\dot{\gamma}^{(q+1)}-\Xi^{(q+1)}\left(\dot{\gamma}, X_{i j}^{(1)}, X_{i j}^{(2)}, R ; T\right) \\
& =\dot{\gamma}^{(q+1)}-\left[Z^{-1}(T)\left(J_{2}^{t r}-3 G \dot{\gamma}^{(q+1)} \Delta t-R^{(q+1)}-k\right)\right]^{n(T)} .
\end{aligned}
$$

The last equation can be solved iteratively using Newton-Raphson's scheme. Thus, an additional incremental step $p$ is introduced. Let's now set at the first iteration $\gamma^{(q+1)(0)}=\gamma^{(q)}, X_{i j}^{(1)^{(q+1)(0)}}=X_{i j}^{(1)^{(q)}}, X_{i j}^{(2)^{(q+1)(0)}}=X_{i j}^{(2)^{(q)}}$ and $R^{(q+1)(0)}=R^{(q)}$. After rearrangement and computing the partial derivatives, one can obtain

$$
\dot{\gamma}^{(q+1)(p+1)}=\dot{\gamma}^{(q+1)(p)}-\frac{1}{\Delta t} \frac{\Omega^{(q+1)(p)} \dot{T}^{(q+1)(p)}+\phi^{(q+1)(p)} \Phi^{(q+1)(p)}}{3 G+\phi^{(q+1)(p)}+\Psi^{(q+1)(p)}},
$$


with

$$
\left\{\begin{array}{l}
\Psi=\sum_{m=1}^{2} v^{(m)}\left(\xi^{(m)}-N_{i j} X_{i j}^{(m)}\right)+\zeta(\kappa-R)+H \\
\phi=Z\left(n Z^{1-n}\left(J_{2}^{t r}-3 G \dot{\gamma}^{(q+1)} \Delta t-R^{(q+1)}-k\right)^{n-1} \Delta t\right)^{-1}, \\
\Omega=\sum_{m=1}^{2}\left(\mathrm{Y}^{(m)}\left(v^{(m)} \xi^{(m)}\right)^{-1} N_{i j} X_{i j}^{(m)}\right)+\left(\gamma H_{, T}+(R-H \gamma)\right) \varrho .
\end{array}\right.
$$

Thus, the viscoplastic multiplier $\gamma$ can be updated at each increment using the following formula

$$
\gamma^{(q+1)(p+1)}=\gamma^{(q+1)(p)}+\dot{\gamma}^{(q+1)(p+1)} \Delta t
$$

The algorithm is assumed to have converged when the convergence criterion is satisfied (i.e. $|\Phi|^{(q+1)(p)}$ is less than a tolerance). Hence, the stresses are computed and updated using Eq. 12 while the viscoplastic strains are found from the following equation

$$
\varepsilon_{i j}^{v p^{(q+1)}}=\varepsilon_{i j}^{v p^{(q)}}+\dot{\gamma}^{(q+1)} N_{i j}^{(q+1)} \Delta t
$$

Using the backward Euler method, the system of equations for the kinematic and isotropic hardening (Eq. 8 and Eq. 11) at time $q+1$ is given as

$$
\begin{gathered}
X_{i j}^{(m)^{(q+1)}}=\frac{X_{i j}^{(m)^{(q)}}+\frac{2}{3} v^{(m)} \xi^{(m)} \dot{\gamma}^{(q+1)} N_{i j}^{(q+1)} \Delta t}{1+v^{(m)} \dot{\gamma}^{(q+1)} \Delta t-\mathrm{Y}^{(m)} \Delta T}, \\
R^{(q+1)}=\frac{R^{(q)}+\zeta\left(\kappa-\zeta^{-1} H\right) \dot{\gamma}^{(q+1)} \Delta t+\left(H_{, T}-H \varrho\right) \gamma^{(q+1)} \Delta T}{1+\zeta \dot{\gamma}^{(q+1)} \Delta t-\varrho \Delta T} .
\end{gathered}
$$

Note that during the FE analysis, ABAQUS carries out the equilibrium iterations while UMAT integrates the material incremental constitutive model. The numerical algorithm for the constitutive law implementation is 


\section{described in Table1.}

Table 1: Return mapping algorithm for the constitutive model

1. At time step $q$ the quantities $\sigma_{i j}^{(q)}, X_{i j}^{(q)}$ and $\gamma^{(q)}$ are known. At time step $q+1$, use the ABAQUS solver and the constitutive relations to identify the total strain tensor $\varepsilon_{i j}^{(q+1)}$.

\section{Elastic prediction.}

Compute the elastic stiffness tensor, trial stresses and trial elastic strains

$$
\begin{gathered}
\sigma_{i j}^{t r}=C_{i j k l}\left(\varepsilon_{i j}^{(q+1)}-\varepsilon_{i j}^{v p^{(q)}}\right) ; \varepsilon_{i j}^{e^{t r}}=\varepsilon_{i j}^{e^{(q)}}+\Delta \varepsilon^{(q+1)} ; \\
\varepsilon_{i j}^{v p^{t r}}=\varepsilon_{i j}^{v p^{(q)}} ; X_{i j}^{(1)(q+1)}=X_{i j}^{(1)(q)} ; X_{i j}^{(2)(q+1)}=X_{i j}^{(2)(q)} ; R^{(q+1)}=R^{(q)} .
\end{gathered}
$$

3. Check the trial yield criterion. Compute the yield function

$$
f^{t r}=J_{2}^{(q+1)}-R^{(q+1)}-k
$$

If $f^{t r} \leq 0$, exit. Accept the predicted $\sigma_{i j}^{t r}, \varepsilon_{i j}^{t r}, \varepsilon_{i j}^{v p^{t r}}, X_{i j}^{(1)^{(q+1)}}, X_{i j}^{(2)^{(q+1)}}$ and $R^{(q+1)}$.

If $f^{t r}>0$, proceed to the next step.

\section{Inelastic correction.}

(a) Initialise

$$
\gamma^{(q+1)(0)}=\gamma^{(q)}, X_{i j}^{(1)^{(q+1)(0)}}=X_{i j}^{(1)^{(q)}}, X_{i j}^{(2)(q+1)(0)}=X_{i j}^{(2)^{(q)}} \text { and } R^{(q+1)(0)}=R^{(q)} .
$$

(b) Compute $\Xi^{(q+1)}$ and solve the residual equation using Newton Raphson's scheme

$$
\Phi^{(q+1)}=\dot{\gamma}^{(q+1)}-\Xi^{(q+1)}\left(\dot{\gamma}, X_{i j}^{(1)}, X_{i j}^{(2)}, R ; T\right) .
$$

(c) Identify $\dot{\gamma}^{(q+1)(p+1)}$ and update $\gamma^{(q+1)(p+1)}$ using Eq.18.

(d) Evaluate $X_{i j}^{(1)^{(q+1)(p+1)}}, X_{i j}^{(2)^{(q+1)(p+1)}}$ and $R^{(q+1)(p+1)}$ using Eq.20 and Eq.21.

(e) If $f^{t r} \leq T O L$, then proceed to step 5. Else set $p=p+1$ and return to (b).

5. Update the elastic strains, the viscoplastic strains and the stresses. 


\section{Determination and calibration of the model parameters}

Note that the main problem encountered when optimizing material parameters is the bounds for material parameters optimised. Hence, allowing a wide range for one set of parameters will fit the experimental data accurately while the obtained parameters could be unrealistic. Thus, a step by step procedure was implemented in order to estimate the initial values of the constants for the viscoplastic model.

The initial guesses of the viscous parameters $(Z$ and $n)$ were determined following the work of Saad [38]. They were estimated using the stress relaxation data in the first cycle from dwell tests carried out at 3 different temperatures (i.e. 400, 550 and $600{ }^{\circ} \mathrm{C}$ ) and based on the assumption that the cyclic hardening is not significantly affecting the viscous constants. Thus, using Eq. 5, the viscous stress can be rearranged as $\log _{10}\left(\sigma_{v}\right)=n^{-1} \log _{10}(\dot{\gamma})+\log _{10}(Z)$ (see. Saad [38]). The total strain rate is then held equal to zero in the stress relaxation period. Thus, the rate of viscoplastic multiplier is determined using the dwell test data (i.e. $\dot{\gamma}=\dot{\varepsilon}_{v p} \approx-\dot{\sigma} / E$, where $E$ stands for the Young's modulus), while the viscous stress is computed using the Cottrel's stress partitioning method (see. Figure 1). Figure 2 shows the plot of $\log _{10}\left(\sigma_{v}\right)$ versus $\log _{10}(\dot{\gamma})$ for the 3 studied temperatures. The viscous constants $Z$ and $n$ are thus identified from the slope of the graph and the y-axis intercept, respectively. It appears from the experimental findings and numerical fitting that the kinematic exponent (resp. viscoplastic resistance) slightly decreases (resp. increases) with increasing temperature.

The initial estimate of isotropic parameters $(\kappa, \zeta$ and $H)$ can be determined from the change in stress amplitude during the cyclic loading at isothermal conditions. The value of $R$ at a certain number of cycles is taken to be the difference between the maximum stress at that cycle and the maximum stress 

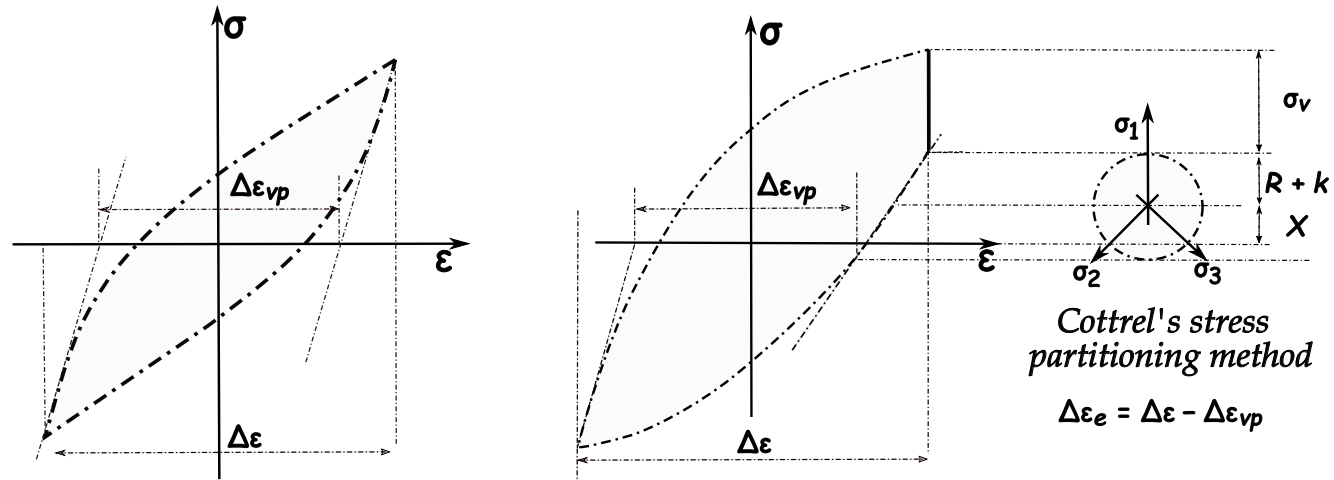

Figure 1: Schematic diagrams of hysteresis loops under tensile-compression tests showing the Cottrel's stress partitioning method.

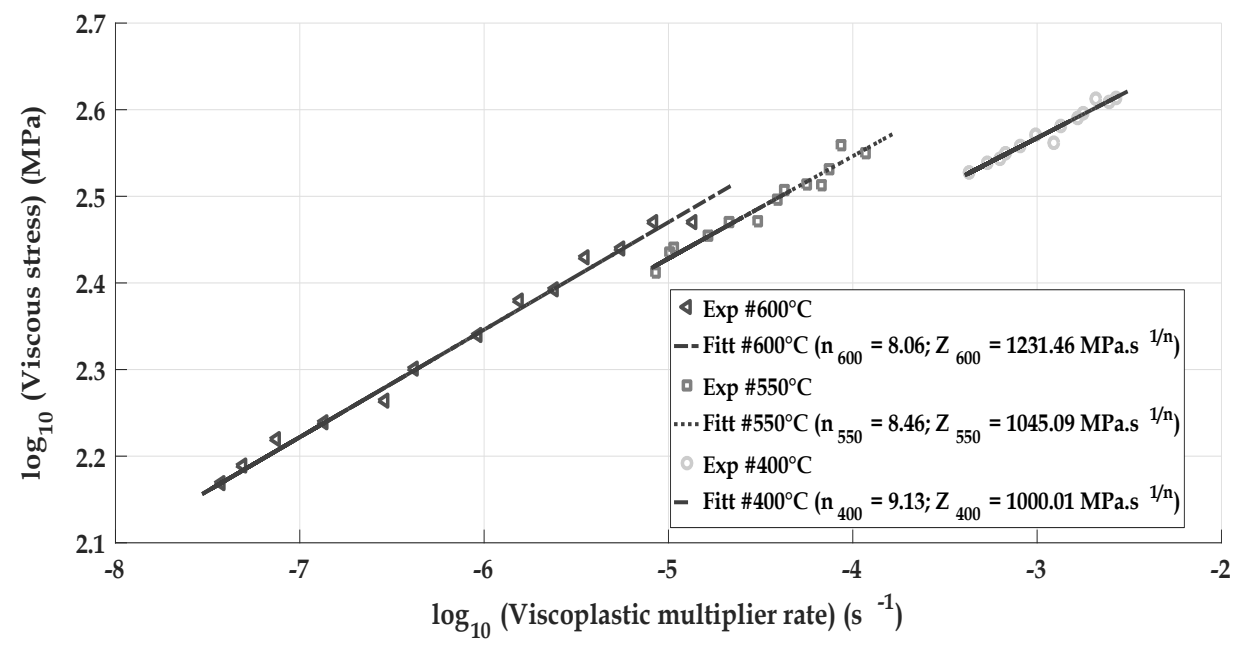

Figure 2: The numerical determination of the viscous parameters at the 3 studied temperatures.

at the first cycle. The integration of Eq. 11 under isothermal conditions can be rearranged in the following form

$$
R(\gamma)=\left(\kappa-H \zeta^{-1}\right)(1-\exp (-\zeta \gamma)),
$$

where $\kappa-H \zeta^{-1}$ is taken to be the maximum stress difference between the first and stabilised cycles (this demonstrates the negative value of $\kappa-$ $H \zeta^{-1}$ ). Note that the viscoplastic multiplier $\gamma$ is considered approximately 


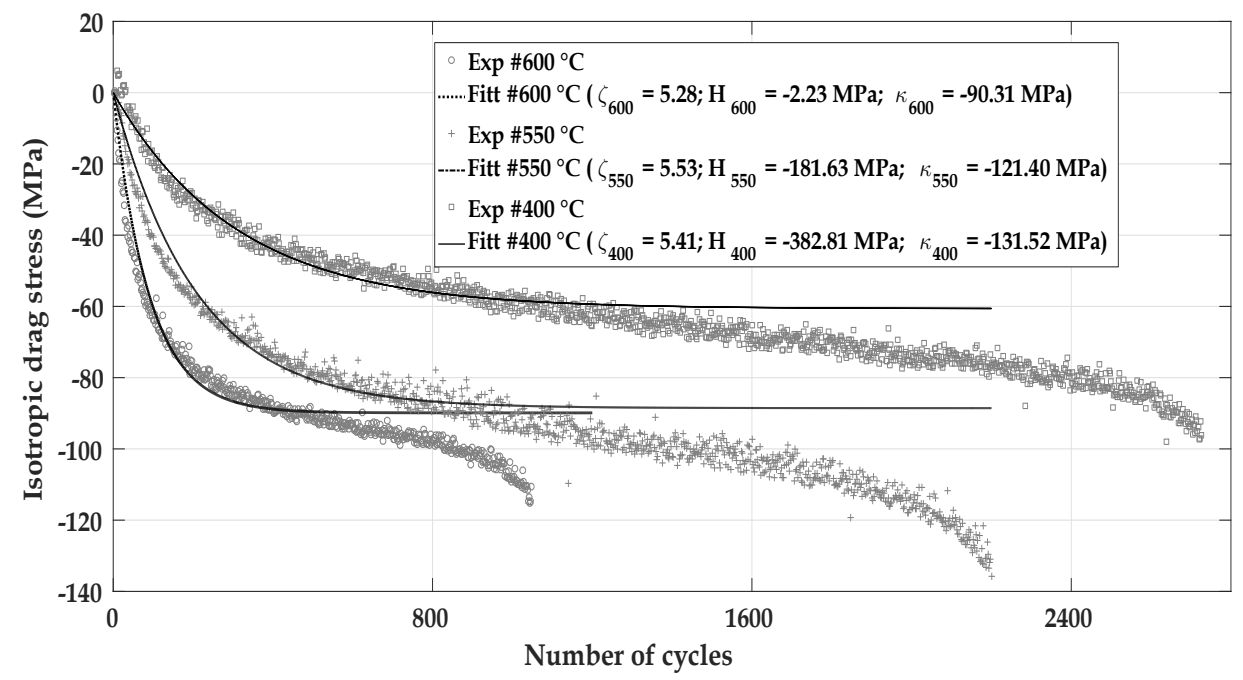

Figure 3: Fitting of change in stress amplitude at isothermal conditions for the determination of $\kappa$, $\zeta$ and $H$.

twice the viscoplastic strain range for each cycle and its value accumulates as cycles increase (i.e. $\gamma \approx 2 N_{c} \Delta \varepsilon_{v p}$, where $N_{c}$ is the number of cycles (see. Chaboche [14])). Thus, the isotropic parameters $(\kappa, \zeta$ and $H)$ are estimated by fitting Eq. 22 to the test data as shown in Figure 3. It can be seen that the parameter $\zeta$ is almost constant for the 3 studied temperatures, while both $\kappa$ and $H$ increase with increasing temperature.

The kinematic hardening parameters were obtained using tension and compression tests from the quasi-stabilised hysteresis loops for different strain amplitudes. In case of isothermal and uniaxial tension-compression tests, the total stress can be written as follows

$$
\sigma=\sum_{m=1,2} \alpha \xi_{m}\left(1-\exp \left(-\alpha v_{m} \varepsilon_{v p}\right)\right)+R+k+\sigma_{v},
$$

where $\alpha= \pm 1$ according to the direction of flow. In Eq. 23 the initial values of backstress and plastic strain, during the analytical integration of Eq. 8, are taken to be equal to zero. Differentiating Eq. 23 with respect to viscoplastic strain $\varepsilon_{v p}$, by assuming that the first component of backstress 
$X_{1}$ has a negligible effect on the hardening of $X_{2}$, and that the yield $k$ and viscous $\sigma_{v}$ stresses are not dependent on $\varepsilon_{v p}$, gives

$$
(\sigma-R)_{, \varepsilon_{v p}}=v_{2} \xi_{2} \exp \left(-\alpha \xi_{2} \varepsilon_{v p}\right) \text {. }
$$

Plotting $\log \left((\sigma-R)_{, \varepsilon_{v p}}\right)$ versus $\varepsilon_{v p}$, and by using data from $X_{2}$ dominated inelastic region, allows the identification of both $v_{2}$ and $\xi_{2}$. Such identification has been carried out for the 3 studied temperatures via isothermal tests (see. Figure $4(\mathrm{a})$ ). When $v_{2}$ and $\xi_{2}$ are estimated, one can differentiate Eq. 23 with respect to viscoplastic strain $\varepsilon_{v p}$ and obtain

$$
\left(\sigma-R-X_{2}\right)_{, \varepsilon_{v p}}=v_{1} \xi_{1} \exp \left(-\alpha \xi_{1} \varepsilon_{v p}\right) .
$$

Thus the hardening constants $v_{1}$ and $\xi_{1}$ can be determined from the $X_{1}$ dominated inelastic region by plotting $\log \left(\left(\sigma-R-X_{2}\right), \varepsilon_{v p}\right)$ versus $\varepsilon_{v p}$ (see. Figure 4(b)). Readers interested in the identification procedure are invited to refer to the work of Saad [38]. It can be seen that $\xi$ decreases with increasing temperature for both kinematic components, while $v_{2}$ holds constant. The values of $v_{1}$ also decrease with increasing temperature. The numerical fitting of the experimental model parameters and after optimisation and validation over wide range of experimental data is summarised in Figure 5 and Table 2. 

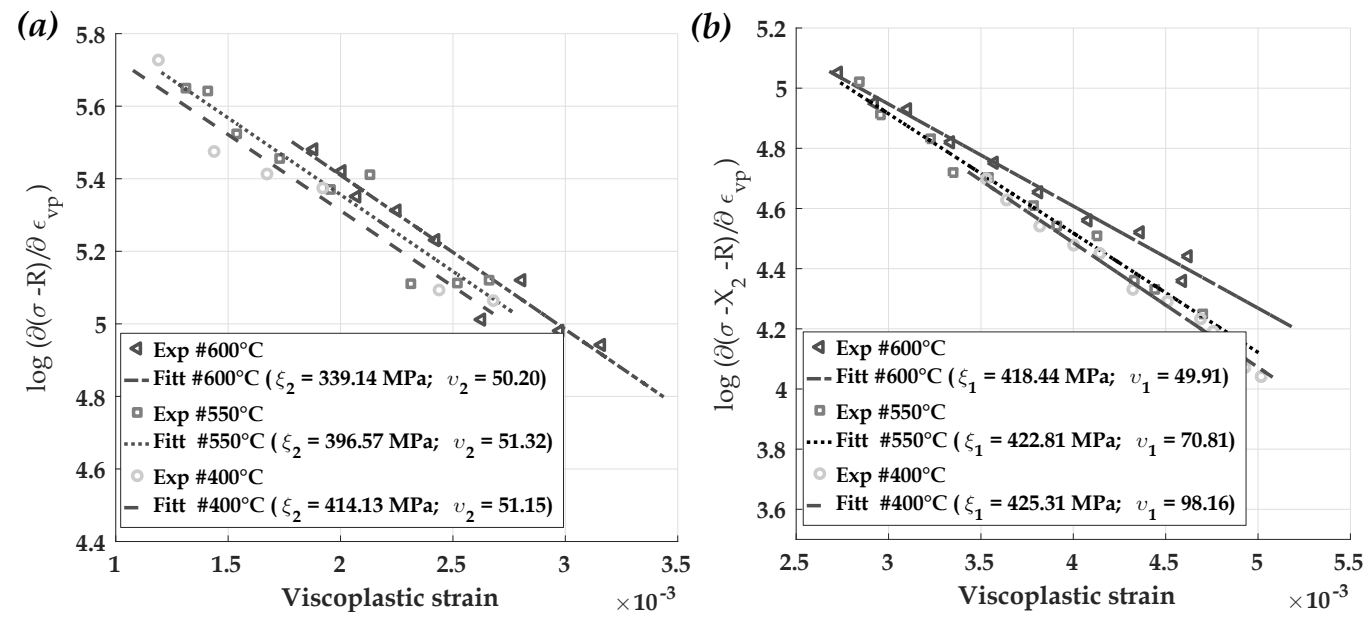

Figure 4: The identification of kinematic hardening parameters for (a) the first and (b) second components at the 3 selected temperatures.
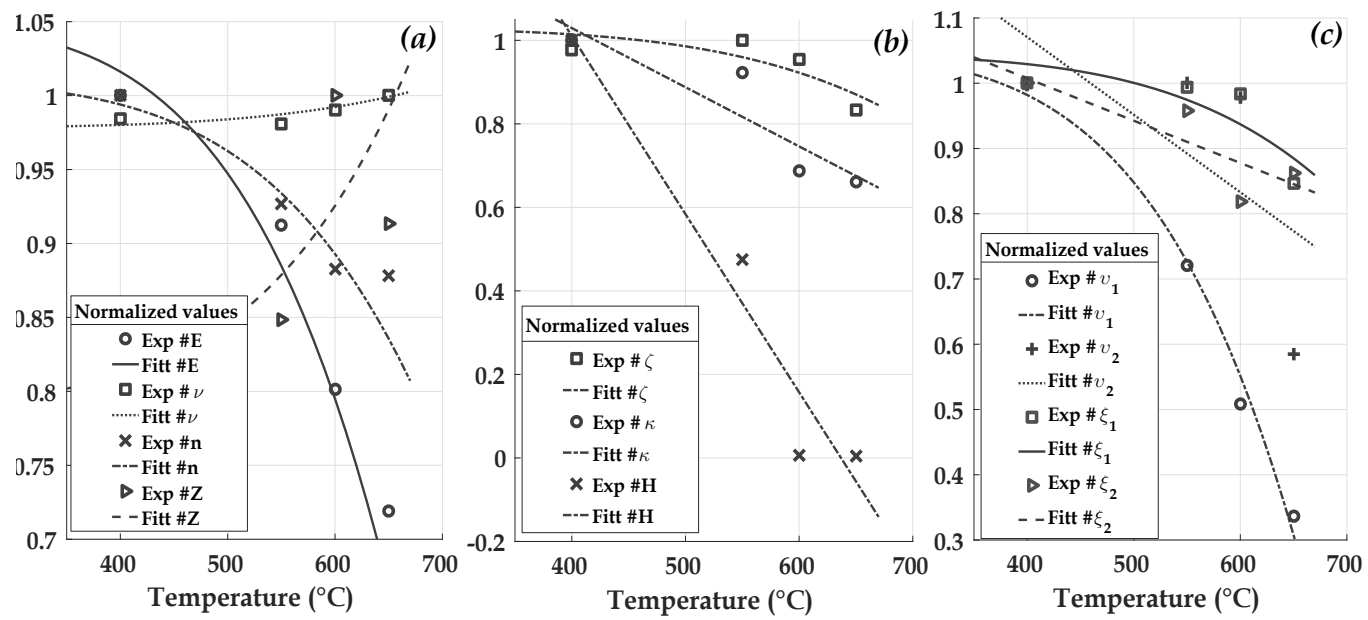

Figure 5: The evolving of model parameters with respect to temperature used for the FE analysis: (a) viscous, (b) isotropic hardening and (c) kinematic hardening parameters. 
Table 2: Identified material parameters in the constitutive model.

\begin{tabular}{lllll}
\hline Mechanical feature & Model parameter & Symbol & Unit & Fitting equation \\
\hline Elasticity & Young Modulus & $E$ & $\mathrm{MPa}$ & $-5.603 .10^{-10} T^{5}+1.795 .10^{5}$ \\
& Poisson's ratio & $v$ & - & $5.632 .10^{-17} T^{5}+0.3072$ \\
Non linear viscosity & Viscoplastic resistance & $Z$ & MPa.s ${ }^{1 / n}$ & $2.099 .10^{-12} T^{5}+976.5$ \\
& Viscous exponent & $n$ & - & $-1.367 .10^{-14} T^{5}+9.215$ \\
& & & & \\
Non linear hardening 1 1 & Hardening modulus & $\xi_{1}$ & $\mathrm{MPa}$ & $-5.802 .10^{-13} T^{5}+443.7$ \\
& Non linear parameter 1 & $v_{1}$ & - & $-6.258 .10^{-13} T^{5}+102.8$ \\
& Hardening modulus 2 & $\xi 2$ & $\mathrm{MPa}$ & $-2.683 .10^{-1} T+524.5$ \\
& Non linear parameter 2 & $v_{2}$ & - & $-6.099 .10^{-2} T+79.34$ \\
& isotropic parameter & $H$ & $\mathrm{MPa}$ & $1.633 T-1040$ \\
& Asymptotic value & $\kappa$ & $\mathrm{MPa}$ & $1.863 .10^{-1} T-209.9$ \\
& Stabilization parameter & $\zeta$ & - & $-7.517 .10^{-15} T^{5}+5.686$ \\
\hline
\end{tabular}

The capability of the proposed model is further checked in the simulation of hysteresis loops. The comparison of the simulated mechanical responses and experimental data at various cycle numbers (initial and half-life cycle hysteresis loops) is shown in Figure 6. It can be seen that the simulated hysteresis loops agree with experimental results at different isothermal conditions. It is also interesting to mention that the current model can approximate the evolving of the cyclic mechanical parameters such as the hysteresis area, the slope and change in stress (see. Figure 6). The associated stress relaxation amounts were also compared with experimental data and show a fairly good agreement between the numerical approximation and experimental finding, especially at the beginning of loading. At enough loading time, the experimental viscous stress becomes underestimated at $400{ }^{\circ} \mathrm{C}$ and $550{ }^{\circ} \mathrm{C}$, while seems to be overestimated at $600{ }^{\circ} \mathrm{C}$.

Note that the material generally exhibits a cyclic softening followed by the failure of the specimen. The onset of the failure is generally attributed to damage processes. The current material does not include the effect of 
fatigue damage and thus cannot reasonably reflect the whole life-fatigue behaviour of the investigated material. However, the model shows in general considerable capability of approximating the temperature dependent cyclic hardening and strain cyclic behaviour under isothermal loading conditions. In order to develop a fully thermomechanical analysis, it is of great importance to take care of heat diffusion processes and estimate heat sources that would involve during the deformation of the investigated material. It is also interesting to mention that all above model predictions are computed under strain-controlled mode with constant strain rates. Thus, it is necessary to inspect the performance of the viscoplastic model under stress-controlled mode where the strain rates would vary with loading. 

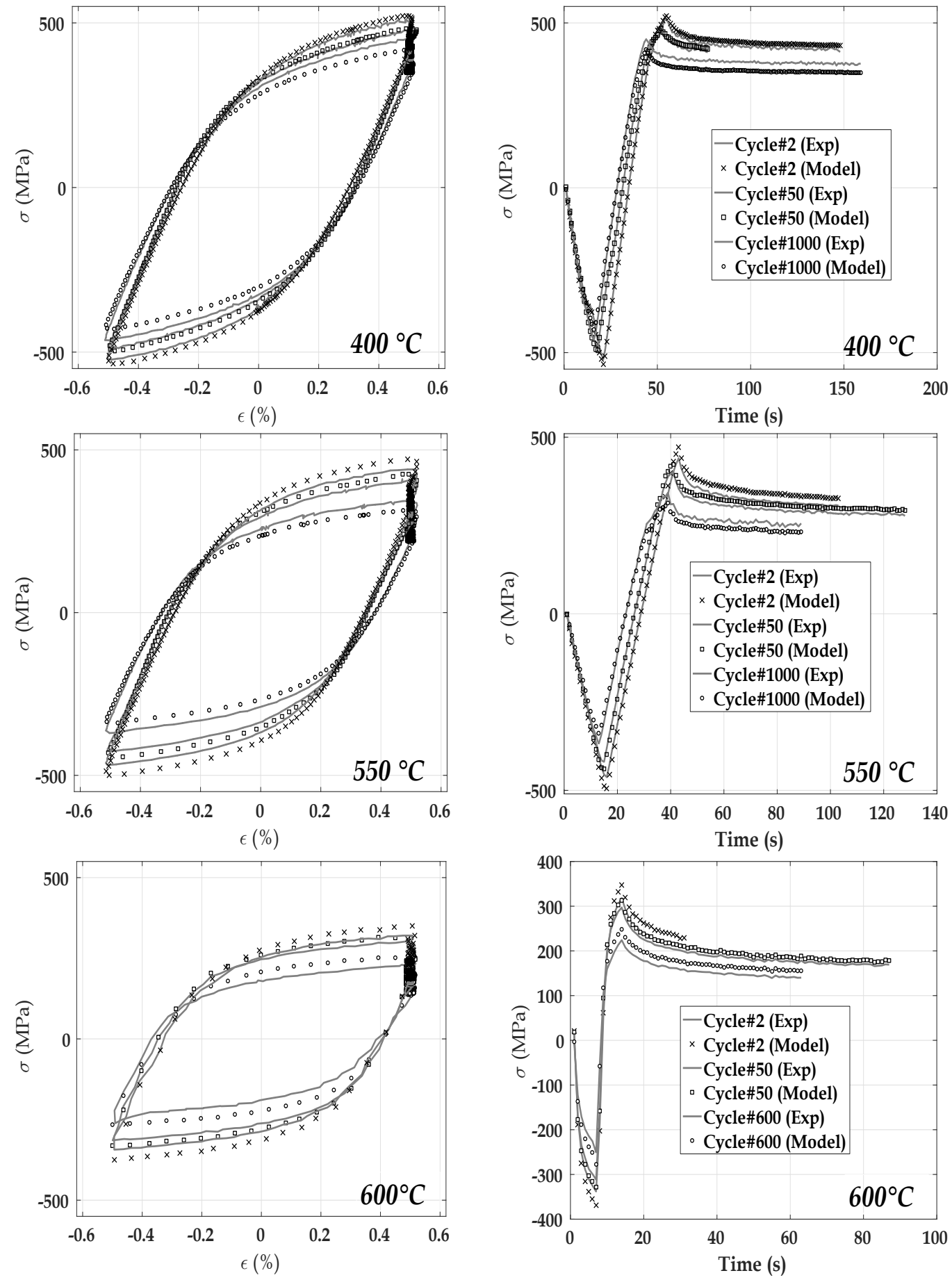

Figure 6: Isothermal hold-time fatigue model predictions for 400,550 and $600{ }^{\circ} \mathrm{C}$ at strain rate of $0.1 \% . s^{-1}$ with 2 mins holds at maximum tension. 


\section{Applications of the constitutive model to the FE Analysis of $300 \mathrm{MW}$ turbine rotor}

Industrial turbines operating in flexible power plants go through frequent daily starts-stops, depending on the market demand. At these severe conditions, a turbine rotor is being continuously cyclically loaded due to the high temperature and mechanical loading, and as a result its life is being reduced. The rotor plays a vital role in a turbine, it spans the whole length of the turbine from inlet to outlet. This creates a large temperature difference in sections along the rotor length. Those turbine parts located in the rotating section are very important as their failure could result in a serious incident causing destructive damage to the turbine and its surroundings. The analysed rotor was taken from a $300 \mathrm{MW}$ gas turbine used in the combined cycle power plant. The rotor was simulated using the fully unified elasto-viscoplastic model presented earlier, it was implemented into the ABAQUS FEA software using the developed UMAT subroutine and the associated material properties as shown in Table 2. It was then used to investigate behaviour of the rotor subjected to creep-fatigue during a number of cycles of start-dwell-stop loading sequence.

The rotor itself is made out of 3 major parts: a low pressure turbine, a high pressure turbine and a compressor. The compressor comprises of 23 rows of blades, a high pressure turbine from single row of blades and a low pressure turbine from 4 rows of blades and 3 rows of heat shields. The extent of the rotor geometry used for the study is shown in the Figure 7. Taking advantage of radial symmetry about the central axis the rotor was simulated as an axisymmetric model. Gas is flowing along its axis from the compressor side toward the turbine at the end. 


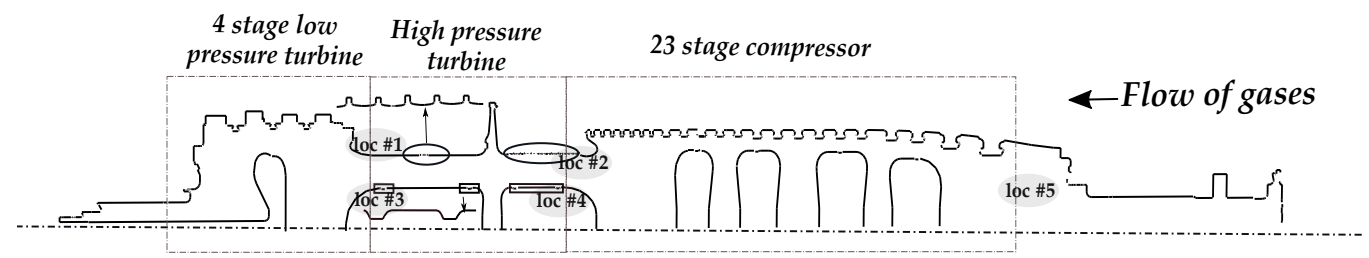

Figure 7: Cross section of the axisymmetric gas turbine rotor model.

The process of the thermal and mechanical analysis of the turbine rotor is as follows:

(i) Construction of the finite element model of the rotor.

(ii) Temperature distribution during the loading is determined during the heat transfer step.

(iii) Temperature data is used together with mechanical loads to simulate the thermal and mechanical stress analysis, which considers viscoplastic material with temperature dependent properties.

\subsection{Thermomechanical loading and boundary conditions}

Figure 8 (a) shows a section of the rotor identifying boundary conditions and loads to which it is exposed during the operation. The mechanical load is composed of the centrifugal force from the blade and rotor gravity. The blades were not modelled explicitly as this would make the model excessively complex and they are not the main focus of this work. Instead an equivalent centrifugal force calculated using blade mass and its centre of gravity was applied to positions on the rotor where the blades would be normally attached. An equivalent pressure load was applied to the blade root groove taking account of the size of the contact surface as shown in Figure 8 (b). The rotor gravity generates centrifugal forces within the rotor itself, and therefore a centrifugal load was applied to the rest of the model using rotational velocity as a variable. The material density of $7750 \mathrm{~kg} / \mathrm{m}^{3}$ was assumed during the simulation. In addition to the mechanical loads, the surface of the rotor is exposed to the hot gases that are the product 
of combustion. A representative spatially and time varying temperature profile was applied to the surface of the rotor during the start-dwell-shut down cycle. Gas temperatures along the turbine have been calculated from a thermo-dynamic assessment and applied as thermal boundary conditions at each stage within the finite element model together with the corresponding heat transfer coefficients. For a cold start a uniform rotor starting temperature profile of $11^{\circ} \mathrm{C}$ was assumed to be conservative. An axial displacement constraint located at the intake end surface of the rotor and central axis of rotation were used to fix the rotor in modelling space.

(a)

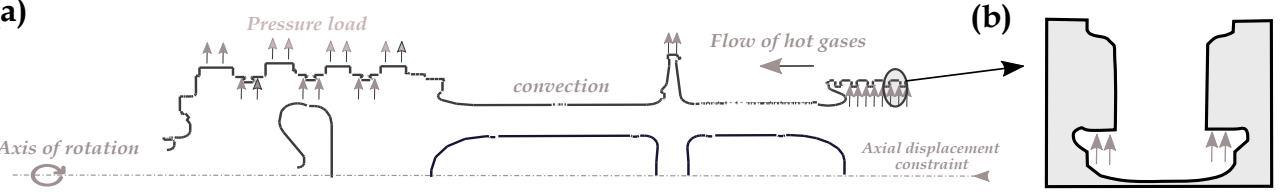

Figure 8: Schematic diagram showing (a) loads and boundary conditions, and (b) blade forces applied within the blade root.

A simplified loading pattern showing variation of the rotational speed and temperature during the cycle is shown in Figure 9. This loading sequence consists of a number of repeating cycles with mechanical and thermal parts. The magnitude of the mechanical loading is controlled by the rotational speed of the rotor. In addition to the mechanical loads, the rotor is also exposed to the hot gas running along its length. The temperature of the gas is not uniform along the rotor length and also fluctuates cyclically according to the loading pattern. Key points in the cycle were used to analyse the results: point $(a)$ is close to the end of the start-up phase when the load is already applied but have not yet reached its peak value, point $(b)$ is located at the end of the dwell period at full load and point (c) is at the end of the second dwell period after unload. The model was taken through 10 start-up and shut-down cycles which allowed steady-creep fatigue cycling to be achieved. The loading cycle consists of two half hour long periods 
of start-up and shutdown with a 12 hours dwell period $t_{1}$ at full power in between, followed by an 11 hours dwell period $t_{2}$ at rest. This represents a typical pattern for high energy demand during the day time, when turbines are turned on and night time, when the demand falls and they are turned off. The peak magnitude of the rotational speed achieved during the 12 hours fully loaded period used to generate the centrifugal load was set at $3000 \mathrm{rpm}$.

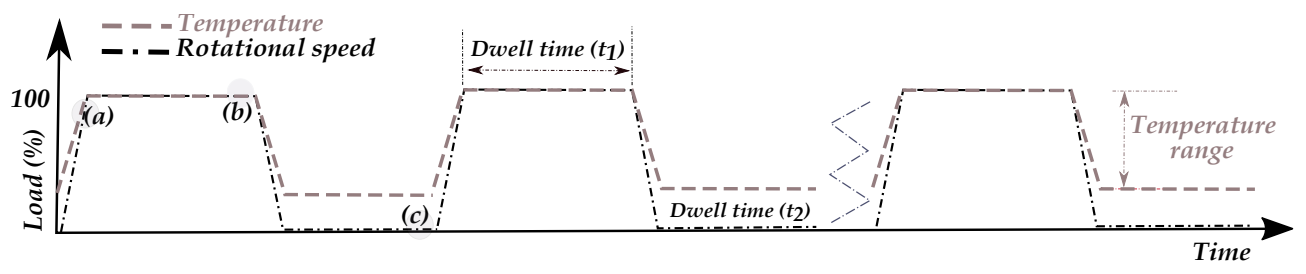

Figure 9: Schematic diagram for intermittent operation showing variation of the rotor outer surface temperature and its rotational speed. Time points $a, b$ and $c$ showing their relative location within the loading pattern.

As sections of the rotor are at different positions along the gas path, the temperature of the environment around them is different. The temperature distribution along the outer surface of the rotor after a period of dwell at full load when temperature has stabilised is shown in Figure 10. The inlet temperature at the compressor was conservatively taken to be $11^{\circ} \mathrm{C}$ and the outlet temperature was calculated to be at $554{ }^{\circ} \mathrm{C}$. In the intermediate stages between inlet and outlet of compressor a linear increase of the temperature is expected. Following the compressor, the temperature of the gas is reduced significantly to about $340{ }^{\circ} \mathrm{C}$, this is due to the addition of the cooling air to the gas stream. From the outlet of the compressor to the inlet of the low pressure turbine there is a steady small increase in the air temperature to about $380^{\circ} \mathrm{C}$. Within the low pressure turbine gas temperature is further reduced by blade and rotor surface cooling to about $184^{\circ} \mathrm{C}$. 


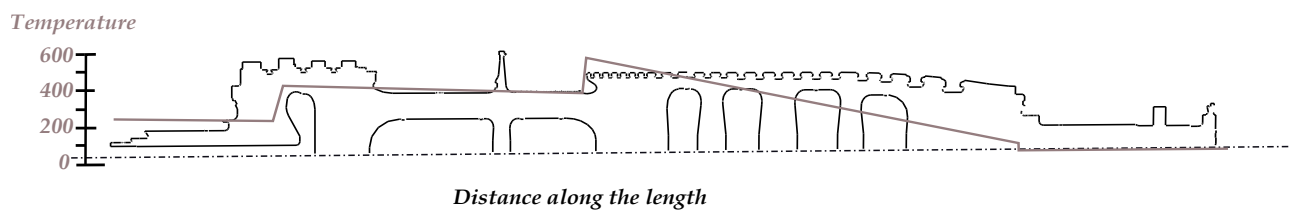

Figure 10: Surface temperature distribution along the rotor length.

A section of the FE model geometry for the rotor is shown in Figure 11 showing meshed detail of the middle section. The same mesh was used both for the heat transfer and stress analyses. Due to presence of small features such as grooves and protrusions that are a part of sealing structure, in such areas refined mesh of smaller element size was placed. There were 33,718 computational elements and 104,667 nodes generated in total. Uncoupled analysis was performed by doing heat transfer analysis first in a steady-state to solve for the uncoupled transient thermal displacements and then static analysis to calculate mechanical displacements. Indeed, a transient thermal analysis was first performed to predict the time varying temperature distribution within the rotor during one start-stop cycle. These time varying temperature distributions were then used to predict stress at various time-steps within each of the 10 structural cycles modelled. For the heat transfer step, 8-node quadratic axisymmetric heat transfer quadrilateral elements DCAX8 were used and for the stress analysis, 8-node biquadratic axisymmetric quadrilateral elements $C A X 8 R$ were used. The heat transfer step is an important step in the simulation, as the accuracy of the thermal results directly affect the mechanical response of the structure. The input data are gas temperature, temperature dependent thermal conductivity, thermal expansion coefficient and specific heat capacity. The material information used in the model was a combination of original forging material data (long term creep tests and tensile tests) and comparison of the material test data held on file. 


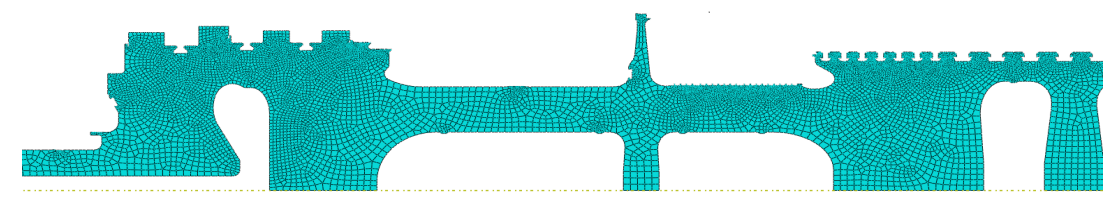

Figure 11: Finite element mesh of the rotor.

\subsection{Anisothermal finite element analysis}

In order to understand the effects of the varying environmental rotor temperature and duty on predicted temperature fields, von Mises stresses and the locations of the peak values are shown in Figure 12. The stress distributions are sampled at critical time points a1-c10 which are shown in Figure 9. We are interested in the spatial and temporal distributions at different points in the cycle and their evolutions as the number of loading cycles progresses. A rainbow style coloured scale with blue for minimum and red for maximum values is used to show the distribution of magnitudes within the geometry. For easier comparison stress contours have been plotted using the same scale with minimum and maximum values fixed. During the first cycle, at the end of start-up at time point a1 as shown in Figure 12 (a1) we can see large spatial heterogeneity in the area between the compressor and low pressure turbine implying a major stress concentration in that area. There are numerous heterogeneities due to the non-uniform geometry and unevenly distributed loading. The highest stress areas are located close to the rotor centreline at the base of the high pressure turbine and first stage of the low pressure turbine. There are also some local high stress concentration points around the low pressure turbine heat shield root base and the base of the heatshield separating 1st and 2nd stage low pressure turbine blades. The high stress in this area is due to thermal loads at that point being supplemented by the pressures produced from the heat shield blade centrifugal force. At the time point b1, at end of the dwell period at full load during the first cycle which is shown in Figure 12 (b1) a small reduction in the stress intensity is occurring due to the relaxation 

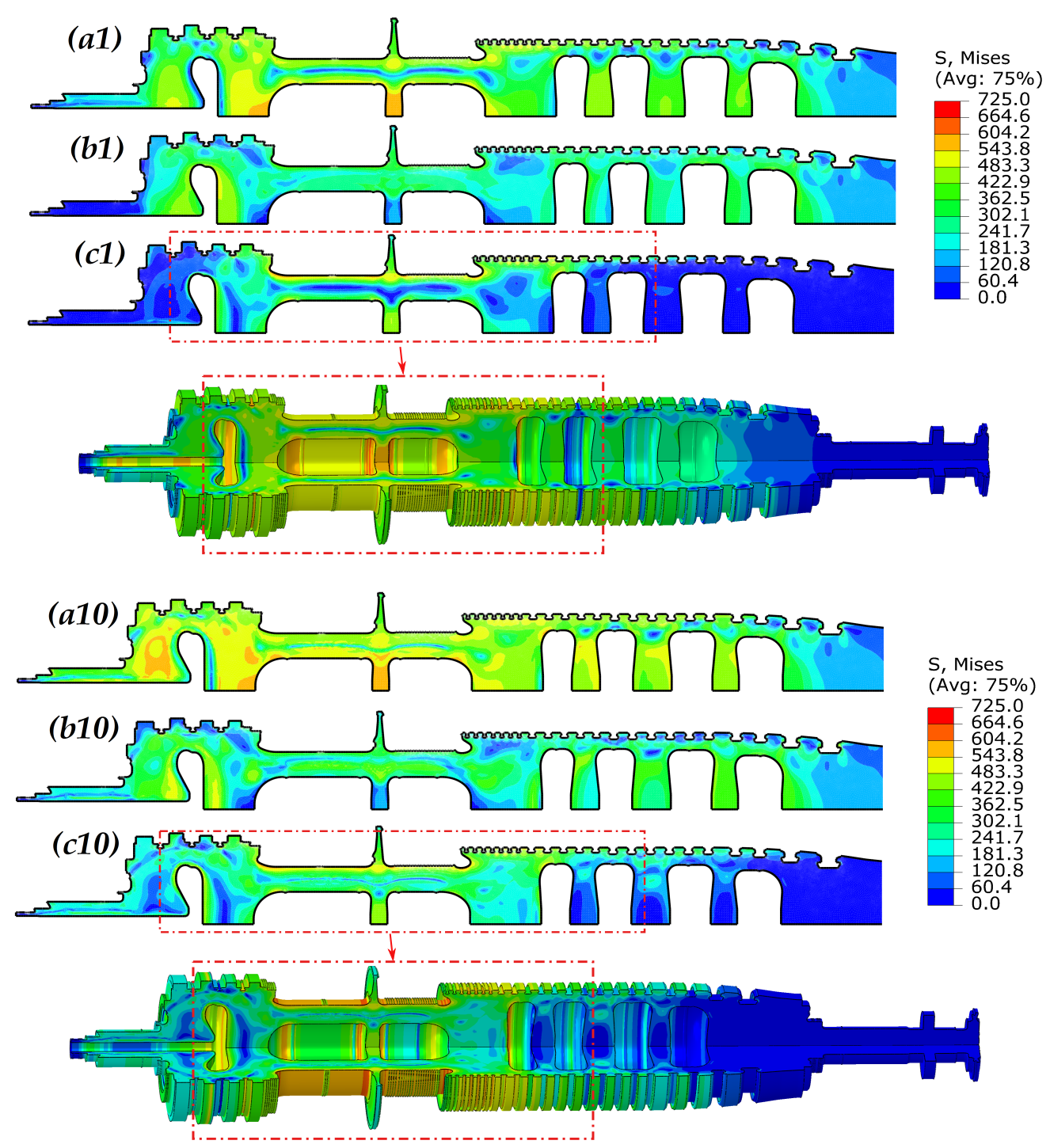

Figure 12: Contour plots of von Mises stress in MPa of rotor at different time points for the first and tenth cycle.

of strain. The main area of high stress has moved towards the inner bore of the low pressure turbine, this end of the rotor has an open ended bore and this gives rise to bending forces in this location. At the time point $\mathrm{c} 1$, at the end of the dwell period after shut down, local residual stresses at 
zero-maintained stress appear in the area between the compressor and low pressure turbine. The majority of spatial heterogeneities are along the outer surface of the rotor and at the base of the high pressure turbine. At time point a10, the stress concentration areas increase in size and become more prominent with more intensity appearing especially in the area of the low pressure turbine. At time point b10, the stress contour follows a similar trend with increase in stress magnitudes and areas when compared to b1, although more local and mostly on the outer surface of the compressor. In case of time point $\mathrm{c} 10$, there is a significant shrinkage of the uniform areas if compared to $\mathrm{c} 1$, this means that the local residual stress is spreading out as the number of cycles increases.

Temperature contours have also been plotted using the same scale for easier comparison with minimum and maximum values fixed. During the first cycle at the end of start-up as is shown in Figure 13 (a1), the surface of the rotor starts to warm up due to the convective heat transfer from the surrounding hot gas, as the temperature of the gas gradually increases. The core of the rotor is still cold at this point leading to stresses due to the temperature up-shock. The heat then gradually moves to the rotor core due to conduction producing circumferential variation in temperature until equilibrium is reached as shown in Figure 13 (b1). The only blue area that is left corresponds to the compressor intake, which is ingesting cold air cooling down that part of the rotor.

In Figure 14 a-e von Mises stresses and temperatures are shown for the first three cycles as they evolve with time, figures a-e correspond to locations 1-5 from Figure 7. For location 1 shown in Figure 14 (a), as it is located on the outer surface it is directly exposed to the gas flow, meaning that its temperature changes rapidly resulting in the square shaped temperature curve. The von Mises stress curve has a large spike to around $510 \mathrm{MPa}$ 

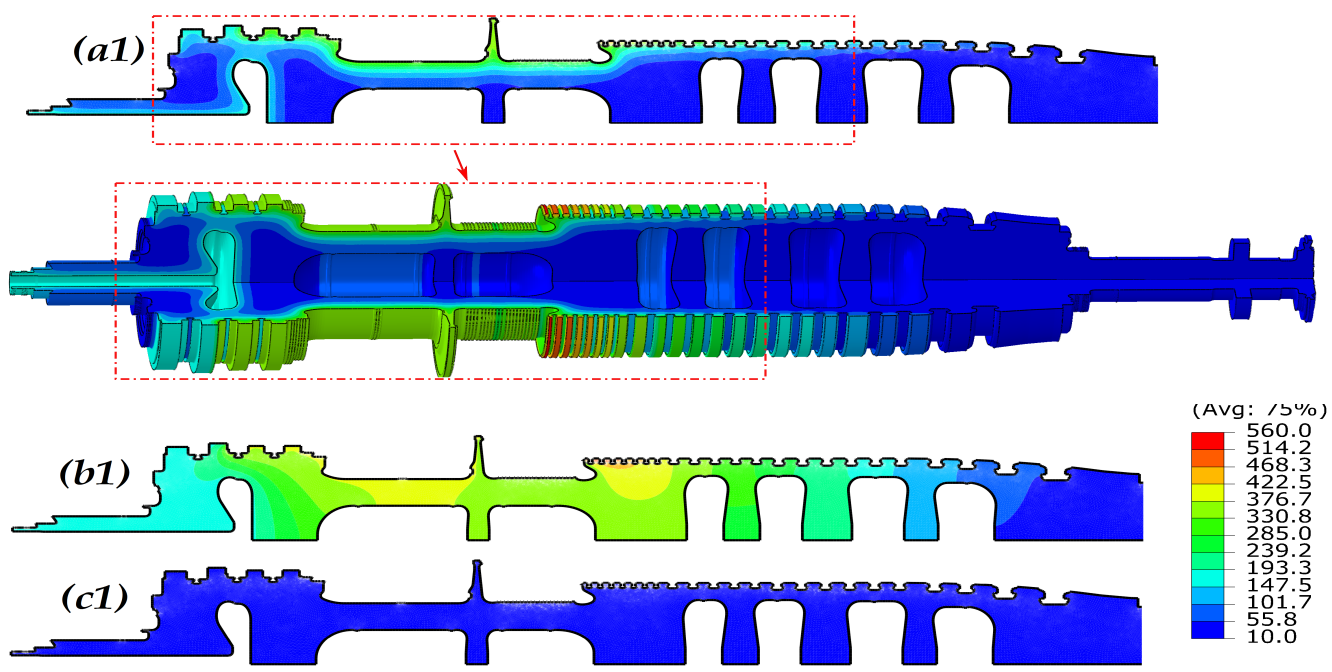

Figure 13: Contour plots of temperature in ${ }^{\circ} \mathrm{C}$ of rotor at different time points for the first cycle.

at the start up as the loading is applied, the stress drops off significantly to around $130 \mathrm{MPa}$ as the turbine enters the dwell period. During the dwell the stress gradually increases eventually stabilising after a couple hours, after which it stays almost constant. A similar behaviour is observed during the shut-down, a large spike is observed around $680 \mathrm{MPa}$ as the load is reduced, which also drops off to around $140 \mathrm{MPa}$ and then gradually reaches around $590 \mathrm{MPa}$ during the dwell period and stays at that level till the end of dwell. Following two cycles show a similar trend with a small variation in the stress levels observed. For location 3 shown in Figure 14 (c), as it is located on the inner part of the rotor, the temperature increases gradually, at a high rate initially during loading and then slower during the dwell period. The shut-down is also followed by a sharp decrease in temperature and then gradually slower during the dwell. Similar spikes in von Mises stress are observed during the start-up and shut-down periods as in the case of locations 1 and 2, although it takes longer for stress to change its magnitude in both load-unload and dwell periods. For location 5 shown in Figure 14 (e), only a small increase in temperature during loading 
is observed, which is expected as it is far from the hot temperature zone. The von Mises stress curve does not have the same spikes present as in the case with the locations 1 to 4 located in the hot area, instead stress increases gradually starting from the beginning of the loading, followed by a smooth gradual decline after the loading is removed.

During the first cycle for location 5 the large temperature change produces significantly larger overall stress due to the cold start condition. Also every cycle is followed by a large clearly visible on the plot peak caused by the sudden drop of temperature and forced cooling followed by a rise in temperature. For the location 5 within the low temperature region maximum thermal stress is about $90 \mathrm{MPa}$ and mechanical stress about $700 \mathrm{MPa}$ due to a large size of the first stage compressor blade, producing stress of about 690 when loads are combined.

Figure 15 shows the distribution of the viscoplastic multiplier in the area of the turbine between the compressor and the low pressure turbine. Four locations are numbered in the figure: location 1 is on the outer surface in the area of low pressure turbine intake, location 2 is at the outer surface of the compressor exit, and locations 3 and 4 are on the inner bore across from locations 1 and 2. A fifth location is taken for reference at the compressor intake, where the variation of the temperature and applied stresses are minimal.

Based on the locations described in Figure 15 the evolution of viscoplastic multiplier with time was plotted in Figure 14 (f). The highest accumulation of plastic strain occurs in location 1 and the lowest in location 5 . In location 5 the plastic strain is much smaller than in the rest of the locations, which can be explained by a small thermal loading at that location. 

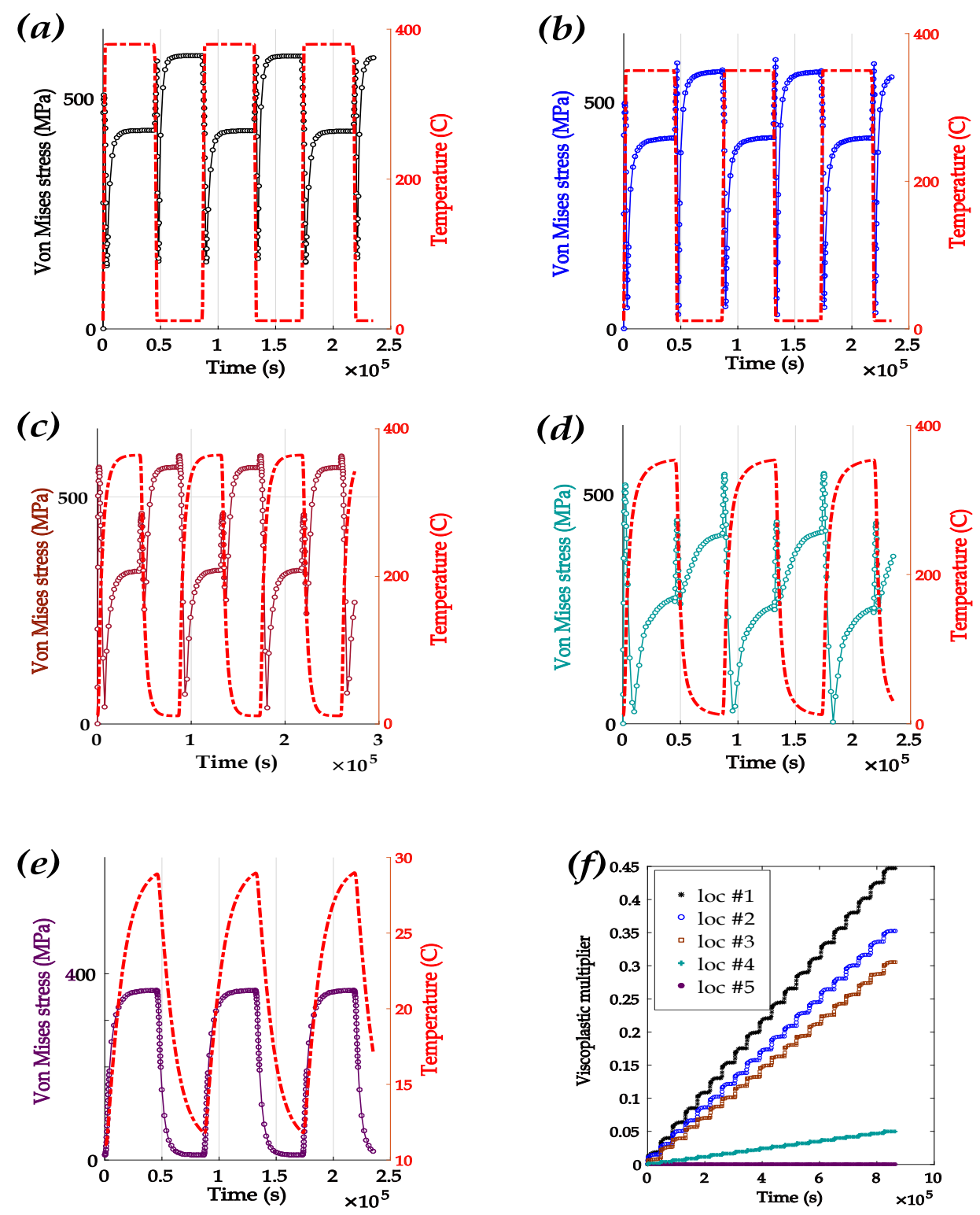

Figure 14: von Mises stress and temperature evolution for the first 3 cycles for locations: (a) 1, (b) 2, (c) 3, (d) 4 and (e) 5. (f) Viscoplastic multiplier evolution for locations 1 to 5.

In Figure 15 the viscoplastic multiplier showing the evolution of plastic strain was plotted on the swept rotor profile for a better visualisation of the critical areas. Cycles 6, 8 and 10 were chosen to show its evolution when its 


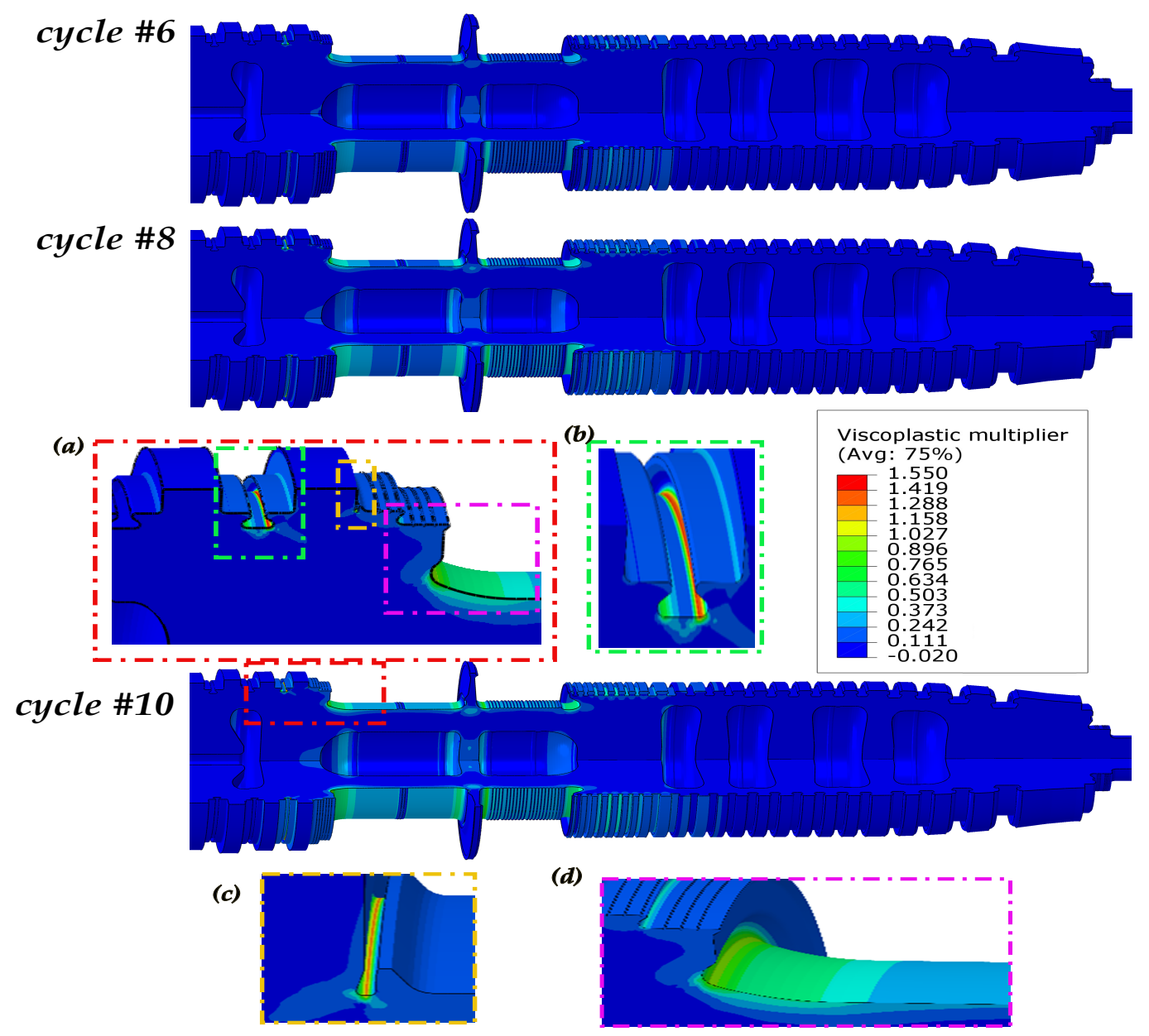

Figure 15: (a) Intake part of the low pressure turbine with stage 1 and 2 row blade attachments. (b) Heat shield root separating stages 1 and 2. (c) Radius within the insert near the low pressure turbine first stage blade. (d) Radius at the low pressure turbine intake.

accumulation becomes the most prominent. At cycle 6 the plastic strain can be seen developing in multiple areas shaped as narrow strips between the last stages of the compressor and the first stages of the low pressure turbine. As we move to cycle 8 the areas affected by plastic strain can be seen widening up as well as increasing in magnitude. By the time we get to cycle 10 the areas affected by plastic strain have widened even further, covering a large area around the high pressure turbine and with new significant areas 
developing, getting as far as stage 12 in the compressor and stage 3 in the low pressure turbine. Now, we will look at how plastic strain has evolved by cycle 10 in more detail, by looking at the details (a-d) of Figure 15. In Figure 15 (a) the locations of highest plastic strain are shown. Figure 15 (b) shows a heat shield root within the low pressure turbine separating stage 1 and 2 row blade attachments. At the base of the root the surface is seen to be red in colour which means that high plastic strain is present. The other two locations were the radius within the insert near the low pressure turbine first stage blade shown in Figure 15 (c) and the radius at the low pressure turbine intake shown in Figure 15 (d).

\section{Conclusions}

The modelling challenge addressed in this article, is to apply a unified viscoplasticity model, capable of simulating a broad set of isothermal lowcycle fatigue and low-cycle fatigue-creep responses of rotor steel. An improved unified material Chaboche and Lemaitre model, which includes both non-linear isotropic and kinematic hardening behaviour as well as viscoplasticity phenomena has been successfully implemented in Abaqus using the UMAT user subroutine. The model was used to describe the LCF at high temperature levels using non-linear FE analysis performed for a realistic rotor axisymmetrically modelled under representative loading conditions. The influence of thermal effects and geometry singularities on the development of residual stresses was studied. A high attention of the study was devoted to the deformation heterogeneities within the rotor structure and also to the critical high stress areas. Multiaxial behaviour of rotor steel was investigated by modelling it under thermo-mechanical cycling conditions. FE analysis was done by looking at temperature contours, von Mises stress and visco-plastic strain. Variation of temperature and stress with respect to time was analysed. Critical zones observed during the analysis were localised. Profiles of the temperature contours have shown large 
temperature differences during the start-up part of the cycle. Within the relatively short period of start-up a rapid increase in the temperature of the surface was observed producing local temperature gradient and leading to a sharp rise in stress. Stresses up to $725 \mathrm{MPa}$ have been generated at various locations mostly at the outer surface of the hot area of the rotor with several locations containing highest von Mises stress concentrations noted as potential crack initiation sites. This analysis indicated that heterogeneities start to appear from the very beginning of mechanical loading and intensify cycle after cycle. These spatial localisations can be linked to damage mechanisms.

Even if the objective of this work is addressed to the rotors of industrial gas turbines, this technology is of general use, providing a good technique for design and for in-service assessment for all components of gas turbines and other equipment under similar working conditions and subjected to creep phenomena and plasticity during each cycle. Rate-dependence essentially reflects the viscosity of the material, which is at the same time closely associated with temperature. Normally it is expected that the material becomes increasingly rate dependent as the temperature increases.

Several problems discussed in paper are highly required to be concerned and resolved in the future work. The potential implications and extensions of such viscoplastic analysis to the power plant life span can be considered. Further research activity will be focused on the performance assessment of rotors containing more than a single material within its body. There is also a demand for a refinement to describe creep characteristics for aged material conditions. The model could be further improved by modifying UMAT and adding damage variable. Also the geometry of the rotor to which the model was applied could be improved by switching from axisymmetric model to a three dimensional model. An axisymmetric model is an acceptable simplification, but it would be beneficial to create a three dimensional 
model to further improve the analysis accuracy by having more accurate modelling of the blade locations.

\section{Acknowledgments}

This work is supported by the Engineering and Physical Sciences Research Council (EPSRC) through the Flex-E-Plant project (Grant number: $\mathrm{EP} / \mathrm{K} 021095 / 1$ ) and the IMPULSE project (grant number EP/N509991/1).

We also thank the following partners for their the valuable contributions: GE Power, Doosan Babcock Limited, Centrica plc., EDF Energy (West Burton Power) Limited, Uniper Technologies Limited, Goodwin Steel Castings Limited, NPL Management Limited, R-MC Power Recovery Limited, RWE Generation UK plc., Scottish and Southern Energy (SSE) plc., Siemens Industrial Turbomachinery and TWI Limited.

\section{References}

[1] Ahmed, R., Barrett, P. R., Hassan, T., 2016. Unified viscoplasticity modeling for isothermal low-cycle fatigue and fatigue-creep stressstrain responses of haynes 230. International Journal of Solids and Structures 88-89, $131-145$.

[2] Ambroziak, A., Klosowski, P., 2006. The elasto-viscoplastic chaboche model. Task Quarterly 10 (1), 49 - 61.

[3] Armstrong, P. J., Frederick, C. O., 1966. A mathematical representation of the multiaxial bauschinger effect. Central Electricity Generating Board, Berkeley Nuclear Laboratories, Research and Development Department, CEGB Report No. RD/B/N 731. 
[4] Arnold, S., Saleeb, A., 1994. On the thermodynamic framework of generalized coupled thermoelastic-viscoplastic-damage modeling. International Journal of Plasticity 10 (3), 263 - 278.

[5] Barrett, R., O'Donoghue, P., Leen, S., 2013. An improved unified viscoplastic constitutive model for strain-rate sensitivity in high temperature fatigue. International Journal of Fatigue 48, 192 - 204.

[6] Barrett, R. A., Farragher, T. P., Hyde, C. J., O'Dowd, N. P., O'Donoghue, P. E., Leen, S. B., 2014. A unified viscoplastic model for high temperature low cycle fatigue of service-aged p91 steel. Journal of Pressure Vessel Technology 136, 021402-021402.

[7] Becker, M., Hackenberg, H.-P., 2011. A constitutive model for rate dependent and rate independent inelasticity. application to in718. International Journal of Plasticity 27 (4), 596-619.

[8] Besson, J., 2010. Continuum models of ductile fracture: A review. International Journal of Damage Mechanics, 19, 3- 52.

[9] Bodner, S., Partom, Y., 1975. Constitutive equations for elasticviscoplastic strain-hardening materials. Journal of Aplied Mechanics $42,385-389$.

[10] Cailletaud, G., Kaczmarek, H., Policella, H., 1984. Some elements on multiaxial behaviour of 3161 stainless steel at room temperature. Mechanics of Materials 3 (4), 333 - 347.

[11] Cailletaud, G., Sai, K., 1995. Study of plastic/viscoplastic models with various inelastic mechanisms. International Journal of Plasticity 11 (8), $991-1005$.

[12] Cernocky, E., Krempl, E., 1980. A theory of thermoviscoplasticity based on infinitesimal total strain. International Journal of Solids and Structures $16(8), 723-741$. 
[13] Chaboche, J., 1977. Viscoplastic constitutive equations for the description of cyclic and anisotropic behaviour of metals. Bull. Acad. Polon. Sci., Ser. Sci. Tech. 25, $33-42$.

[14] Chaboche, J., 2008. A review of some plasticity and viscoplasticity constitutive theories. International Journal of Plasticity 24 (10), 1642 1693, special Issue in Honor of Jean-Louis Chaboche.

[15] Chaboche, J., Nouailhas, D., 1989. A unified constitutive model for cyclic viscoplasticity and its applications to various stainless steels. ASME. J. Eng. Mater. Technol. 111, $424-430$.

[16] Chaboche, J., Rousselier, G., 1983. On the plastic and viscoplastic constitutive equations - part i: Rules developed with internal variable concept. ASME. J. Pressure Vessel Technol. 105, 153 - 158.

[17] Chaboche, J. L., Dang Van, K., Cordier, G., 1979. Modelization of the strain memory effect on the cyclic hardening of 316 stainless steel. ONERA, TP no. 1979-109, L 11/3.

[18] Chan, K. S., Lindholm, U. S., Bodner, S. R., Walker, K. P., 1989. High temperature inelastic deformation under uniaxial loading: Theory and experiment. ASME. Journal of Engineering Materials and Technology $111,345-353$.

[19] Contesti, E., Cailletaud, G., 1987. Description of creep-plasticity interaction with non-unified constitutive equations: Application to an austenitic stainless steel. 6th International seminar on inelastic analysis and life prediction in high temperature environment.

[20] Corum, J., Blass, J., 1991. Rules for design of alloy 617 nuclear components to very high temperatures. Fatigue, Fracture, and Risk 215, $147-153$. 
[21] Freed, A. D., Chaboche, J. L., Walker, K. P., Mar 1991. A viscoplastic theory with thermodynamic considerations. Acta Mechanica 90 (1), 155-174.

[22] Freed, A. D., Walker, K. P., 1993. Viscoplasticity with creep and plasticity bounds. International Journal of Plasticity 9 (2), 213 - 242.

[23] Germain, P., Nguyen, Q., Suquet, P., 1983. Continuum thermomechanics. Journal of Applied Mechanics 50, 1010 - 1020.

[24] Johnson, G. R., Cook, W. H., 1983. A constitutive model and data for metals subjected to large strains, high strain rates and high temperatures. Proceeding of the 7th International Symposium on Ballistics, The Hague, The Netherlands, $541-547$.

[25] Kawai, M., Ohashi, Y., 1987. Coupled effect between creep and plasticity of type 316 stainless steel at elevated temperature. 2nd international conference on constitutive laws for engineering materials: Theory and applications. Tucson, Arizona. DESAI et al. (eds.), Elsevier.

[26] Kocks, U., Argon, A., Ashby, M., 1975. Thermodynamics and Kinetics of Slip. No. 19 in International Series of Monographs in Natural Philosophy. Pergamon Press.

[27] Krempl, E., 2000. Viscoplastic models for high temperature applications. International Journal of Solids and Structures, 37 (1), 279 291.

[28] Kyaw, S. T., Rouse, J. P., Lu, J., Sun, W., 2016. Determination of material parameters for a unified viscoplasticity-damage model for a p91 power plant steel. International Journal of Mechanical Sciences 115, 168 - 179.

[29] Miller, A., 1976. An inelastic constitutive model for monotonic, cyclic, and creep deformation: Part i - equations development and analytical 
procedures. ASME. Journal of Engineering Materials and Technology 98, $97-105$.

[30] Nayebi, A., Ranjbar, H., Rokhgireh, H., 2012. Analysis of unified continuum damage mechanics model of gas turbine rotor steel: life assessment. Journal of Materials Design and Applications 227 (3), 216 -225 .

[31] Ohno, N., 1990. Recent topics in constitutive modeling of cyclic plasticity and viscoplasticity. ASME. Applied Mechanics Review 43, $283-$ 295.

[32] Ohno, N., Wang, J.-D., 1993. Kinematic hardening rules with critical state of dynamic recovery, part i: formulation and basic features for ratchetting behavior. International Journal of Plasticity 9 (3), 375 - 390.

[33] Ohno, N., Wang, J.-D., 1993. Kinematic hardening rules with critical state of dynamic recovery, part ii: Application to experiments of ratchetting behavior. International Journal of Plasticity 9 (3), $391-403$.

[34] Olszak, W., Perzyna, P., 1969. On thermal effects in viscoplasticity. Zeitschrift für angewandte Mathematik und Physik ZAMP 20 (5), 676-680.

[35] Perzyna, P., 1966. Fundamental problems in viscoplasticity. Advances in Applied Mechanics 9, 243 - 377.

[36] Prager, W., 1949. Recent developments in the mathematical theory of plasticity. Journal of Applied Physics, 20 (235).

[37] Rao, K. B. S., Meurer, H.-P., Schuster, H., 1988. Creep-fatigue interaction of inconel 617 at 950c in simulated nuclear reactor helium. Materials Science and Engineering: A 104, 37 - 51. 
[38] Saad, A., 2012. Cyclic plasticity and creep of power plant materials. Ph.D. thesis, University of Nottingham.

[39] Saad, A., Sun, W., Tobi, A., 2016. Multiaxial viscoplasticity modelling of power plant steel. Key Engineering Materials 701, 230-234.

[40] Sellars, C. M., McTegart, W., 1966. On the mechanism of hot deformation. Acta Metallurgica 14 (9), 1136 - 1138.

[41] Shutov, A., Kreibig, R., 2008. Finite strain viscoplasticity with nonlinear kinematic hardening: Phenomenological modeling and time integration. Computer Methods in Applied Mechanics and Engineering 197 (21), 2015 - 2029.

[42] Simo, J., Hughes, T., 1998. Computational inelasticity. Springer-Verlag, NY.

[43] Tong, J., Zhan, Z.-L., Vermeulen, B., 2004. Modelling of cyclic plasticity and viscoplasticity of a nickel-based alloy using chaboche constitutive equations. International Journal of Fatigue 26 (8), 829 - 837.

[44] Velay, V., Bernhart, G., Penazzi, L., 2006. Cyclic behavior modeling of a tempered martensitic hot work tool steel. International Journal of Plasticity, 22 (3), $459-496$.

[45] Wang, W., Buhl, P., Klenk, A., 2014. A unified viscoplastic constitutive model with damage for multi-axial creep-fatigue loading. International Journal of Damage Mechanics 24, 363 - 382.

[46] Wang, W., Buhl, P., Klenk, A., Liu, Y., 2016. Study of creep-fatigue behavior in a $1000 \mathrm{mw}$ rotor using a unified viscoplastic constitutive model with damage. International Journal of Damage Mechanics 25 (2), $178-202$. 
[47] Zhan, Z.-L., Tong, J., 2007. A study of cyclic plasticity and viscoplasticity in a new nickel-based superalloy using unified constitutive equations. part ii: Simulation of cyclic stress relaxation. Mechanics of Materials 39 (1), 73 - 80.

[48] Zhang, S.-L., Xuan, F.-Z., 2017. Interaction of cyclic softening and stress relaxation of $9-12 \%$ cr steel under strain-controlled fatiguecreep condition: Experimental and modeling. International Journal of Plasticity 98, $45-64$.

[49] Zhang, Z., Delagnes, D., Bernhart, G., 2002. Anisothermal cyclic plasticity modelling of martensitic steels. International Journal of Fatigue $24(6), 635-648$.

[50] Zienkiewicz, O., Cormeau, I., 1974. Visco-plasticity - plasticity and creep in elastic solids - a unified numerical solution approach. Numerical Methods in emgineering 8, $821-845$. 